\title{
A DIRECT PROOF THAT A LINEARLY ORDERED SPACE IS HEREDITARILY COLLECTIONWISE NORMAL ${ }^{1}$
}

\section{LYNN A. STEEN}

Although it appears well known that a linearly ordered space is completely normal (=hereditarily normal), most available proofs (in, for instance, [1] and [2]) are very indirect. In this paper we present a direct proof of a stronger theorem, namely that the interval topology is hereditarily collectionwise normal. ${ }^{2}$

If $X$ is linearly ordered, we will call a set $S \subset X$ convex if $a, b \in S$ and $a<t<b$ implies $t \in S$. The union of any collection of convex sets with nonempty intersection is convex, so any subset $S$ of $X$ can be uniquely expressed as a union of disjoint maximal convex sets called convex components. Clearly every interval in $X$ is convex but not conversely, and we will, as usual, denote intervals by $(a, b),(a, b],[a, b)$, or $[a, b]$. In what follows, $X$ will denote a linearly ordered space, i.e., a linearly ordered set endowed with the usual open interval topology.

Suppose $\left\{A_{i}\right\}$ is a discrete family of subsets of $X$. Let

$$
A_{i}^{*}=\bigcup\left\{[a, b] \mid a, b \in A_{i},[a, b] \cap A_{j}=\varnothing \forall j \neq i\right\} .
$$

Then $A_{i} \subset A_{i}{ }^{*}$, and $A_{i}{ }^{*} \cap A_{j}{ }^{*}=\varnothing$ whenever $i \neq j$; in fact, the family $\left\{A_{i}^{*}\right\}$ is discrete. To prove this, we select for each $x \in X$ a neighborhood $I_{x}$ which intersects at most one of the sets $A_{i}$. If $I_{x}$ meets exactly one element of $\left\{A_{i}\right\}$, say $A_{k}$, and if $x$ is not an endpoint of $X$, we can take $I_{x}$ to be an interval $(s, t)$. Then if $i \neq k,(s, t)$ may intersect $A_{i}{ }^{*}$ only if it intersects some interval $[a, b]$ where $a, b \in A_{i}$. But since $(s, t) \cap A_{i}=\varnothing$ and $a, b \in A_{i}$ then $(s, t) \subset(a, b)$ which would imply that $A_{k} \cap A_{i}{ }^{*} \neq \varnothing$. But this is impossible if $i \neq k$, so in this case $I_{x}$ can intersect at most one of the sets $A_{i}{ }^{*}$. Other cases are treated analogously, so $\left\{A_{i}{ }^{*}\right\}$ (and consequently $\operatorname{cl}\left(A_{i}^{*}\right)$ ) is discrete.

If we now write each $A_{i}{ }^{*}$ and $\left(\mathrm{U}_{i} A_{i}{ }^{*}\right)^{\prime}$ as the union of convex components, $A_{i}{ }^{*}=\mathrm{U}_{\alpha} A_{\alpha}^{i}$, and $\left(\bigcup_{i} A_{i}{ }^{*}\right)^{\prime}=\bigcup_{\gamma} C_{\gamma}$, the collection $M=\left\{A_{\alpha}^{i}, C_{\gamma}\right\}$ inherits a linear order from $X$ and is thus itself a linearly ordered set. We claim that in the ordered set $M$, each of the sets $A_{\alpha}^{i}$ has an immediate successor whenever $A_{\alpha}^{i}$ intersects the closure of $S_{\alpha}^{i}$, the set of strict upper bounds for $A_{\alpha}^{i}$. For suppose $A_{\alpha}^{i} \cap S_{\alpha}^{i} \neq \varnothing$. Then $A_{\alpha}^{i} \cap \operatorname{cl}\left(S_{\alpha}^{i}\right)$

Received by the editors December 2, 1968.

1 This work was partially supported by the Research Corporation.

2 The author wishes to thank the referee for several clarifying suggestions. 
contains precisely one point, say $p$, every neighborhood of which intersects $A_{i}{ }^{*}$. Thus since $\operatorname{cl}\left(A_{i}{ }^{*}\right)$ is discrete, there exists a neighborhood $(x, y)$ of $p$ disjoint from $\bigcup_{j \neq i} \operatorname{cl}\left(A_{j}^{*}\right)$. Then $(x, y) \cap S_{\alpha}^{i} \neq \varnothing$, so $(p, y) \neq \varnothing$. But the definition of $A_{i}{ }^{*}$ insures that $(p, y)$ is disjoint from both $A_{i}{ }^{*}$ and $U_{i \neq j} A_{j}{ }^{*}$, so there must exist some set $C_{\gamma}$ containing $(p, y)$. In the linear order on $M, C_{\gamma}$ is the immediate successor to $A_{\alpha}^{i}$, and we will call it $C_{\alpha}^{i+}$.

For each $\gamma$, select and fix some point $k_{\gamma} \in C_{\gamma}$. Then whenever $A_{\alpha}^{i} \cap \operatorname{cl}\left(S_{\alpha}^{i} \neq \varnothing\right.$, there exists a unique $k_{\alpha}^{i+} \in C_{\alpha}^{i+}$, the immediate successor of $A_{\alpha}^{i}$. In such cases, let $I_{\alpha}=\left[p, k_{\alpha}^{i+}\right)$ where $p \in A_{\alpha}^{i} \cap \operatorname{cl}\left(S_{\alpha}^{i}\right)$; otherwise, if $A_{\alpha}^{i} \cap \operatorname{cl}\left(S_{\alpha}^{i}=\varnothing\right.$, let $I_{\alpha}^{i}=\varnothing$. Define $J_{\alpha}^{i}$ similarly for the strict lower bounds of $A_{\alpha}^{i}$ (using the same collection of points $k_{\gamma} \in C_{\gamma}$ ). Then for each $\alpha$ and each $i$, let $U_{\alpha}^{i}=J_{\alpha}^{i} \cup A_{\alpha}^{i} \cup I_{\alpha}^{i}$. Each $U_{\alpha}^{i}$ is clearly an open set containing $A_{\alpha}^{i}$, so $U_{i}=\bigcup_{\alpha} U_{\alpha}^{i}$ is an open set containing $A_{i}{ }^{*}$. Since no $A_{\alpha}^{i}$ intersects any $A_{\beta}^{j}$ for $i \neq j$, and since the use of the same $k_{\gamma}$ throughout implies that no $J_{\alpha}^{i}$ or $I_{\alpha}^{i}$ may intersect any $J_{\beta}^{j}$ or $I_{\beta}^{j}$, it is clear that no $U_{\alpha}^{i}$ can intersect any $U_{\beta}^{j}$ for $i \neq j$. Thus $U_{i} \cap U_{j}=\varnothing$ whenever $i \neq j$, and hence $X$ is collectionwise normal.

Now every subspace of $X$ inherits both a topology as well as a linear order; these need not be compatible, even for open subspaces. (The open subspace $\{\alpha+1 \mid \alpha$ is a limit ordinal $\}$ of the linearly ordered ordinal space $\{\gamma \mid \gamma<\Omega\}$ inherits the discrete topology but is of the same order type as the countable ordinals.) However, the two structures are compatible on convex subspaces of $X$, whence convex subspaces of $X$ are collectionwise normal. Therefore any open subset of $X$ -being the disjoint union of open collectionwise normal subspaces (namely its convex components)-is collectionwise normal. This suffices to prove that every subset $S$ of $X$ is collectionwise normal, since if $\left\{A_{i}\right\}$ is a discrete family in $S$, then each point $s \in S$ has a neighborhood $U_{i} \cap S$ which meets at most one of the sets $A_{i}$. But then $U=U_{8} U_{8}$ is an open set with the same property, and since $U$ is collectionwise normal, so must be $S$. Hence $X$ is hereditarily collectionwise normal.

That $X$ is completely normal (i.e., hereditarily normal) follows as a corollary. But it also may be proved more directly by a slight modification of the proof that $X$ is collectionwise normal.

\section{REFERENCES}

1. E. Cech, Topological spaces, Publ. House Czech. Acad. Sci., Prague, 1959; English Transl., Wiley, New York, 1966. MR 21 \#2962; MR 35 \#2254.

2. S. Gaal, Point set topology, Academic Press, New York, 1964. MR 30 \#1484.

St. Olaf College 